\title{
Very-high-energy gamma-ray emission from the direction of the Galactic globular cluster Terzan 5
}

H.E.S.S. Collaboration, A. Abramowski ${ }^{1}$, F. Acero ${ }^{2}$, F. Aharonian ${ }^{3,4,5}$, A. G. Akhperjanian ${ }^{6,5}$, G. Anton ${ }^{7}$, A. Balzer ${ }^{7}$, A. Barnacka ${ }^{8,9}$, U. Barres de Almeida ${ }^{10, \star}$, Y. Becherini ${ }^{11,12}$, J. Becker ${ }^{13}$, B. Behera ${ }^{14}, K$. Bernlöhr ${ }^{3,15}$, A. Bochow ${ }^{3}$, C. Boisson ${ }^{16}$, J. Bolmont ${ }^{17}$, P. Bordas ${ }^{18}$, J. Brucker ${ }^{7}$, F. Brun ${ }^{12}$, P. Brun ${ }^{9}$, T. Bulik ${ }^{19}$, I. Büsching ${ }^{20,13}$, S. Carrigan ${ }^{3}$, S. Casanova ${ }^{13}$, M. Cerruti ${ }^{16}$, P. M. Chadwick ${ }^{10}$, A. Charbonnier ${ }^{17}$, R. C. G. Chaves ${ }^{3}$, A. Cheesebrough ${ }^{10}$,

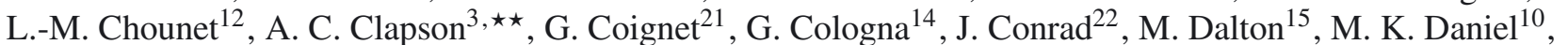
I. D. Davids ${ }^{23}$, B. Degrange ${ }^{12}$, C. Deil ${ }^{3}$, H. J. Dickinson ${ }^{22}$, A. Djannati-Ataï ${ }^{11}$, W. Domainko ${ }^{3}$, L. O'C. Drury ${ }^{4}$, F. Dubois ${ }^{21}$, G. Dubus ${ }^{24}$, K. Dutson ${ }^{25}$, J. Dyks ${ }^{8}$, M. Dyrda ${ }^{26}$, K. Egberts ${ }^{27}$, P. Eger ${ }^{7}$, P. Espigat ${ }^{11}$, L. Fallon ${ }^{4}$, C. Farnier $^{2}$, S. Fegan ${ }^{12}$, F. Feinstein ${ }^{2}$, M. V. Fernandes ${ }^{1}$, A. Fiasson ${ }^{21}$, G. Fontaine ${ }^{12}$, A. Förster ${ }^{3}$, M. Füßling ${ }^{15}$, Y. A. Gallant ${ }^{2}$, H. Gast ${ }^{3}$, L. Gérard ${ }^{11}$, D. Gerbig ${ }^{13}$, B. Giebels ${ }^{12}$, J. F. Glicenstein ${ }^{9}$, B. Glück ${ }^{7}$, P. Goret ${ }^{9}$, D. Göring ${ }^{7}$, S. Häffner ${ }^{7}$, J. D. Hague ${ }^{3}$, D. Hampf ${ }^{1}$, M. Hauser ${ }^{14}$, S. Heinz ${ }^{7}$, G. Heinzelmann ${ }^{1}$, G. Henri' ${ }^{24}$, G. Hermann ${ }^{3}$, J. A. Hinton ${ }^{25}$, A. Hoffmann ${ }^{18}$, W. Hofmann ${ }^{3}$, P. Hofverberg ${ }^{3}$, M. Holler ${ }^{7}$, D. Horns ${ }^{1}$, A. Jacholkowska ${ }^{17}$, O. C. de Jager ${ }^{20}$, C. Jahn ${ }^{7}$, M. Jamrozy ${ }^{28}$, I. Jung ${ }^{7}$, M. A. Kastendieck ${ }^{1}$, K. Katarzyński ${ }^{29}$, U. Katz ${ }^{7}$, S. Kaufmann ${ }^{14}$, D. Keogh ${ }^{10}$, D. Khangulyan ${ }^{3}$, B. Khélifi ${ }^{12}$, D. Klochkov ${ }^{18}$, W. Kluźniak ${ }^{8}$, T. Kneiske ${ }^{1}$, Nu. Komin ${ }^{21}$, K. Kosack ${ }^{9}$, R. Kossakowski ${ }^{21}$, H. Laffon ${ }^{12}$, G. Lamanna ${ }^{21}$, D. Lennarz ${ }^{3}$, T. Lohse ${ }^{15}$, A. Lopatin ${ }^{7}$, C.-C. Lu ${ }^{3}$, V. Marandon ${ }^{11}$, A. Marcowith ${ }^{2}$, J. Masbou ${ }^{21}$, D. Maurin ${ }^{17}$, N. Maxted ${ }^{30}$, T. J. L. McComb ${ }^{10}$, M. C. Medina ${ }^{9}$, J. Méhault ${ }^{2}$, N. Nguyen ${ }^{1}$,

R. Moderski ${ }^{8}$, E. Moulin ${ }^{9}$, C. L. Naumann ${ }^{17}$, M. Naumann-Godo ${ }^{9}$, M. de Naurois ${ }^{12}$, D. Nedbal ${ }^{31}$, D. Nekrassov ${ }^{3}$, B. Nicholas ${ }^{30}$, J. Niemiec ${ }^{26}$, S. J. Nolan ${ }^{10}$, S. Ohm ${ }^{32,25,3}$, E. de Oña Wilhelmi ${ }^{3}$, B. Opitz ${ }^{1}$, M. Ostrowski ${ }^{28}$, I. Oya ${ }^{15}$, M. Panter ${ }^{3}$, M. Paz Arribas ${ }^{15}$, G. Pedaletti ${ }^{14}$, G. Pelletier ${ }^{24}$, P.-O. Petrucci ${ }^{24}$, S. Pita ${ }^{11}$, G. Pühlhofer ${ }^{18}$, M. Punch ${ }^{11}$, A. Quirrenbach ${ }^{14}$, M. Raue ${ }^{1}$, S. M. Rayner ${ }^{10}$, A. Reimer ${ }^{27}$, O. Reimer ${ }^{27}$, M. Renaud ${ }^{2}$, R. de los Reyes ${ }^{3}$, F. Rieger $^{3,33}$, J. Ripken ${ }^{22}$, L. Rob ${ }^{31}$, S. Rosier-Lees ${ }^{21}$, G. Rowell ${ }^{30}$, B. Rudak ${ }^{8}$, C.B. Rulten ${ }^{10}$, J. Ruppel ${ }^{13}$, F. Ryde ${ }^{34}$, V. Sahakian ${ }^{6,5}$, A. Santangelo ${ }^{18}$, R. Schlickeiser ${ }^{13}$, F. M. Schöck ${ }^{7}$, A. Schulz ${ }^{7}$, U. Schwanke ${ }^{15}$, S. Schwarzburg ${ }^{18}$, S. Schwemmer ${ }^{14}$,

M. Sikora ${ }^{8}$, J. L. Skilton ${ }^{32}$, H. Sol ${ }^{16}$, G. Spengler ${ }^{15}$, Ł. Stawarz $^{28}$, R. Steenkamp ${ }^{23}$, C. Stegmann ${ }^{7}$, F. Stinzing ${ }^{7}$,

K. Stycz ${ }^{7}$, I. Sushch ${ }^{15, \star \star \star}$, A. Szostek ${ }^{28}$, J.-P. Tavernet ${ }^{17}$, R. Terrier ${ }^{11}$, M. Tluczykont ${ }^{1}$, K. Valerius ${ }^{7}$, C. van Eldik ${ }^{3}$, G. Vasileiadis ${ }^{2}$, C. Venter ${ }^{20}$, J. P. Vialle ${ }^{21}$, A. Viana 9 , P. Vincent ${ }^{17}$, H. J. Völk ${ }^{3}$, F. Volpe ${ }^{3}$, S. Vorobiov ${ }^{2}$, M. Vorster $^{20}$, S. J. Wagner ${ }^{14}$, M. Ward ${ }^{10}$, R. White ${ }^{25}$, A. Wierzcholska ${ }^{28}$, M. Zacharias ${ }^{13}$, A. Zajczyk ${ }^{8,2}$,

A. A. Zdziarski ${ }^{8}$, A. Zech ${ }^{16}$, and H.-S. Zechlin ${ }^{1}$

(Affiliations can be found after the references)

Received 2 May 2011 / Accepted 16 June 2011

\section{ABSTRACT}

The HESS very-high-energy (VHE, $E>0.1 \mathrm{TeV}$ ) gamma-ray telescope system has discovered a new source, HESS J1747-248. The measured integral flux is $(1.2 \pm 0.3) \times 10^{-12} \mathrm{~cm}^{-2} \mathrm{~s}^{-1}$ above $440 \mathrm{GeV}$ for a power-law photon spectral index of $2.5 \pm 0.3_{\text {stat }} \pm 0.2_{\text {sys. }}$. The VHE gamma-ray source is located in the close vicinity of the Galactic globular cluster Terzan 5 and extends beyond the HESS point spread function $\left(0.07^{\circ}\right)$. The probability of a chance coincidence with Terzan 5 and an unrelated VHE source is quite low $\left(\sim 10^{-4}\right)$. With the largest population of identified millisecond pulsars (msPSRs), a very high core stellar density and the brightest GeV range flux as measured by Fermi-LAT, Terzan 5 stands out among Galactic globular clusters. The properties of the VHE source are briefly discussed in the context of potential emission mechanisms, notably in relation to msPSRs. Interpretation of the available data accommodates several possible origins for this VHE gamma-ray source, although none of them offers a satisfying explanation of its peculiar morphology.

Key words. globular clusters: individual: Terzan 5 - radiation mechanisms: non-thermal - pulsars: general - gamma rays: general

\section{Introduction}

Several types of Galactic VHE $\gamma$-ray sources, such as pulsar wind nebulae (PWNe) and supernova remnants, have been

\footnotetext{
^ Supported by CAPES Foundation, Ministry of Education of Brazil.

$\star \star$ Corresponding author.

$\star \star \star$ Supported by Erasmus Mundus, External Cooperation Window.
}

detected to date, but so far none are in the vicinity of a globular cluster (GC). GCs are very old stellar systems with exceptionally high densities of stars in their cores, leading to numerous stellar collisions (see e.g. Pooley \& Hut 2006), and they also contain many millisecond pulsars (msPSRs, Ransom 2008), likely related to the large number of binary stellar members (Camilo \& Rasio 2005). GCs are predicted to emit VHE $\gamma$-rays 


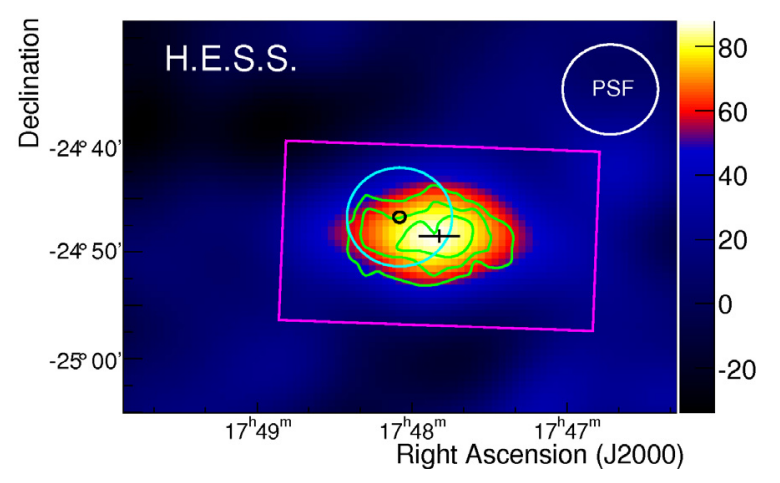

Fig. 1. Exposure-corrected excess image from the HESS data, smoothed with a Gaussian function of width $0.1^{\circ}$ and overlaid with significance contours $(4-6 \sigma)$ in RADec J2000 coordinates. The circles show the half-mass radius (in black) and the larger tidal radius (in cyan) of the GC. The cross indicates the best-fit source position of HESS J1747248 , assuming a 2D Gaussian shape, with $1 \sigma$ uncertainty on each axis. The rectangle represents the integration region used for the full-source spectral analysis. The upper-right corner circle illustrates the instrumental PSF.

from inverse Compton (IC) up-scattering of photons from both stellar radiation fields and the cosmic microwave background by energetic electrons. Electrons could be produced at least at two different sites: in the magnetosphere of the msPSRs (Venter et al. 2009, hereafter VJ09) or at msPSR wind nebulae shocks where electrons produced by the msPSRs (Bednarek \& Sitarek 2007, hereafter BS07) could be re-accelerated. Recently observed GeV $\gamma$-ray emission from several GCs (Fermi-LAT observations: Abdo et al. 2009; Kong et al. 2010; Abdo et al. 2010a; Tam et al. 2011) prove that electrons accelerated up to the required energy range are present in GCs.

This paper reports the discovery of VHE $\gamma$-rays from the direction of the GC Terzan 5. Terzan 5 hosts the largest population of msPSRs detected in a GC so far (33, Ransom 2008). It also contains the largest estimated number of msPSRs as derived from its emission in the $\mathrm{GeV}\left(180_{-90}^{+100}\right.$, Abdo et al. 2010a) and radio domains (60-200, Fruchter \& Goss 2000; Kong et al. 2010). It is located at a distance of $5.9 \mathrm{kpc}$ (Ferraro et al. 2009) at RA(J2000) $17^{\mathrm{h}} 48^{\mathrm{m}} 04^{\mathrm{s}} .85$ and Dec $-24^{\circ} 46^{\prime} 44^{\prime \prime} 6$ (Galactic coordinates: $\left.l=3.84^{\circ}, b=1.69^{\circ}\right)$ and exhibits a core radius $r_{\mathrm{c}}=00^{\prime} 15$, a half-mass radius $r_{\mathrm{h}}=0.52$, and a tidal radius $r_{\mathrm{t}}=4.6$ (Lanzoni et al. 2010). The detection of diffuse non-thermal X-ray emission centred on the GC but extending beyond $r_{\mathrm{h}}$ (between 1 and $5.8 r_{\mathrm{h}}$ ) has been reported by Eger et al. (2010). Observations of other GCs in the VHE domain so far have only resulted in upper limits (on M 13, M 15, M 5, and 47 Tucanae in Anderhub et al. 2009; McCutcheon 2009; Aharonian et al. 2009, respectively, and references therein for older results).

\section{Observation and analysis}

HESS is an array of four Imaging Atmospheric Cherenkov Telescopes, located in the Khomas Highland of Namibia (latitude $23.16^{\circ}$ South, altitude $1800 \mathrm{~m}$ ). Stereoscopic trigger and analysis methods allow efficient background (cosmic ray, CR) rejection and accurate reconstruction of energy (better than 20\%) and arrival direction (better than $0.1^{\circ}$ per event) for $\gamma$-rays in the range $0.1-100 \mathrm{TeV}$. For point-like sources, the array has a nominal detection sensitivity of $\sim 1 \%$ of the flux of the Crab Nebula (Crab) above $1 \mathrm{TeV}$ with a significance of $5 \sigma$ in $25 \mathrm{~h}$ of observation at small zenith angles $\left(<20^{\circ}\right)$. A thorough discussion of the HESS standard analysis based on Hillas parameters and the performance of the instrument can be found in Aharonian et al. (2006). Data on Terzan 5 was obtained by HESS from 2004 to 2010 both as part of its systematic survey of the Galactic plane and with dedicated observations.

For source detection, the data quality cuts result in $90 \mathrm{~h}$ of 3 - and 4-telescope data with an average zenith angle of $20.4^{\circ}$ and a mean pointing direction offset from Terzan 5 of $0.95^{\circ}$. After applying hard cuts (see Aharonian et al. 2006) optimized for point-like sources, with a corresponding energy threshold of $380 \mathrm{GeV}$ and a point spread function (PSF) $68 \%$ containment radius $\mathrm{r}_{\mathrm{PSF}}=0.07^{\circ}\left(4^{\prime} .2\right)$, a source of VHE $\gamma$-rays is detected (see Fig. 1, produced using the template background estimation method, described in Rowell 2003). The significance reaches $5.3 \sigma$ at the position of Terzan 5 with a nearby peak significance of $7.5 \sigma$ pre-trial (above $5 \sigma$ post-trial). All results are confirmed by analysis based on fully independent calibration and analysis chains (de Naurois \& Rolland 2009).

The source appears to extend beyond the GC tidal radius. A 2D Gaussian fit $^{1}$ results in a best-fit position $\left(\chi^{2} / \mathrm{ndf}=0.18\right)$ $\mathrm{RA}(\mathrm{J} 2000) 17^{\mathrm{h}} 47^{\mathrm{m}} 49^{\mathrm{s}} \pm 1^{\mathrm{m}} \mathrm{8}_{\text {stat }} \pm 1^{\mathrm{s}} \cdot 3_{\text {sys }}$ and Dec $-24^{\circ} 48^{\prime} 30^{\prime \prime} \pm$ $36^{\prime \prime}{ }_{\text {stat }} \pm 20^{\prime \prime}$ sys (see Acero et al. 2010a), offset by 4.'0 \pm 1 .'9 from the GC centre, therefore, this new VHE $\gamma$-ray source is named HESS J1747-248. The size of the source is given by the Gaussian widths 9'. $6 \pm 2.4$ and $1.8 \pm 1.2$ for the major and minor axes, respectively, oriented $92^{\circ} \pm 6^{\circ}$ westwards from north.

To establish the photon spectrum of the source, a more restrictive data selection is applied, to improve the energy reconstruction, resulting in a total of $62 \mathrm{~h}$ of live time. Figure 2 illustrates the results of a spectral analysis with the reflected background estimation method (Berge et al. 2007). The test region (shown in Fig. 1) is a rectangle defined from the 2D Gaussian fit (half dimensions $r_{\mathrm{PSF}}+\sigma_{\mathrm{x}}, 2 \times r_{\mathrm{PSF}}$, rotated and centred on the best-fit position). Larger integration regions give lower signal significance. For a power-law spectral model $k\left(\frac{E}{E_{0}}\right)^{-\Gamma}$, the flux normalization $k$ at $1 \mathrm{TeV}$ is $(5.2 \pm 1.1) \times 10^{-13} \mathrm{~cm}^{-2} \mathrm{~s}^{-1} \mathrm{TeV}^{-1}$, and the spectral index $\Gamma=2.5 \pm 0.3_{\text {stat }} \pm 0.2_{\text {sys }}$, corresponding to an integral photon flux within the integration region of $(1.2 \pm 0.3) \times 10^{-12} \mathrm{~cm}^{-2} \mathrm{~s}^{-1}$, or $1.5 \%$ of the Crab flux, in the $0.44-24 \mathrm{TeV}$ range $\left(\chi^{2} / \mathrm{ndf}=1.1\right)$. There are not enough excess events to discuss a more complex spectral model.

For comparison with the VHE $\gamma$-ray flux upper limit on the GC 47 Tucanae (Aharonian et al. 2009), see Sect. 3.3, where a spectral analysis for a point-like source centred on the core of Terzan 5 is carried out. It results in a compatible photon index and a reduced flux normalization, corresponding to an integral flux in the energy range $(0.8<E<48.6 \mathrm{TeV})$ of $(1.9 \pm 0.7) \times$ $10^{-13} \mathrm{~cm}^{-2} \mathrm{~s}^{-1}$.

\section{Discussion}

In the vicinity of the discovered VHE $\gamma$-ray source, the archival multi-wavelength (MWL) data and astronomical catalogues do not show any typical candidate VHE $\gamma$-ray emitter (e.g. an SNR, a young energetic pulsar or PWN, see Clapson et al. 2011). Its properties are unexpected for a GC, notably its extension, indication of offset $(\sim 2.0 \sigma$ level) from Terzan 5 and misalignment with the centre of the GC. Thus the source is challenging to interpret.

Several approaches to constrain possible origins of the VHE radiation are considered here. First, chance coincidence probabilities, notably with young unrelated PWNe, are derived in Sect. 3.1. Next, the possible emission mechanisms for GCs are addressed. VHE leptons are covered in Sect. 3.2 in relation to

1 Obtained with the data analysis pipeline CIAO v4.2 sherpa v2 (Freeman et al. 2001): http://cxc.cfa.harvard.edu/sherpa/ 


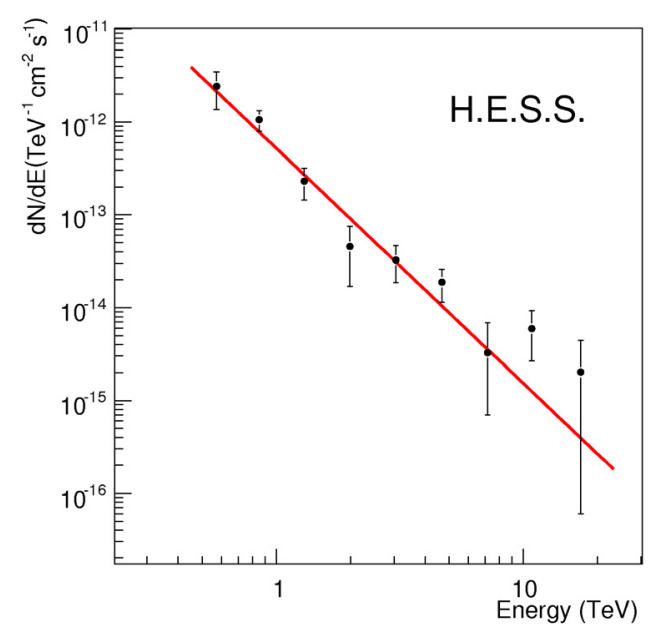

Fig. 2. VHE $\gamma$-ray spectrum of HESS J1747-248 with $1 \sigma$ error bars, fitted with a power-law model. The fit results are discussed in the text.

msPSRs. A comparison with other GCs, identified as potential VHE $\gamma$-ray sources, is performed in Sect. 3.3. Finally, possibilities for a hadronic emission scenario are discussed in Sect. 3.4.

\subsection{Chance coincidence}

The proximity on the sky of the GC and the VHE $\gamma$-ray source could simply be the chance coincidence of two physically unrelated objects. In particular, the source parameters (extension, photon spectrum) are compatible with those of identified VHE PWNe (see e.g. Mattana et al. 2009). The probabilities of a chance coincidence based on the distribution of known objects are considered in two ways.

The probability of source confusion in the VHE domain can be derived from the distribution of source counts as a function of Galactic latitude. Chaves et al. (2009) find that this distribution, in the longitude range $-85^{\circ}<l<60^{\circ}$, can be described by a Gaussian profile (containing 48 sources) centred at $b=-0.26^{\circ}$ with a width $\sigma=0.40^{\circ}$ and additionally four outliers at latitude $b<-2^{\circ}$, below the Galactic plane. There are no other detected VHE $\gamma$-ray sources in the latitude band $1.5^{\circ}<b<2^{\circ}$. At a latitude of $1.7^{\circ}$, Terzan 5 is almost $5 \sigma$ away from the centre of the Gaussian, in a region where the total number of expected sources is negligible $\left(\lessgtr 10^{-3}\right)$; i.e. the source presented here would be an extreme outlier in the Gaussian distribution. Using one source as the total expectation for this latitude band over the longitude range observed by the HESS Galactic Plane Survey, the probability of finding a VHE $\gamma$-ray source within a conservative distance of $0.1^{\circ}$ from Terzan 5 is estimated to be $\sim 10^{-4}$. A caveat to this argument is that the sky coverage by HESS is highly nonuniform, with longer exposure (favouring source detection) in regions typically located very close to the Galactic plane.

A connection has been suggested between VHE $\gamma$-ray sources and the PWN of pulsars with distance-scaled spin-down luminosity above $10^{34} \mathrm{erg} \mathrm{s}^{-1} \mathrm{kpc}^{-2}$ (Carrigan et al. 2008). They could be observable during certain stages of their evolution with characteristics similar to the detected VHE source (Mattana et al. 2009). In the band defined above, there is only one such pulsar (PSR J1124-5916) in the ATNF catalogue ${ }^{2}$ (Manchester et al. 2005). It has been proposed that the number of radio-loud and radio-quiet pulsars might be comparable (e.g. Gonthier et al. 2004), therefore, one may expect a single undetected radio-quiet powerful pulsar. The resulting probability of chance coincidence

\footnotetext{
2 http://www.atnf.csiro.au/research/pulsar/psrcat/ (version 1.40).
}

with the VHE $\gamma$-ray source within the rectangular region shown in Fig. 1 is again $\sim 10^{-4}$. This approach is limited by the strength of the association (not every powerful pulsar is associated with a VHE $\gamma$-ray source) and by the biases and incompleteness of the catalogues (e.g. Gonthier et al. 2004), illustrated by the discovery of radio-quiet $\mathrm{GeV} \gamma$-ray pulsars (Abdo et al. 2010b, but none so far in the neighbourhood of Terzan 5).

In summary, it is unlikely that the association of the VHE $\gamma$-ray source and the GC is by chance, but due to simplifications in the probability estimation, such a possibility cannot firmly be excluded.

\subsection{Leptonic VHE $\gamma$-ray production}

Owing to the highly energetic electrons produced by their msPSR populations, GCs could be $\gamma$-ray emitters, as predicted by three different models. In the GeV range, VJ09 explore the $\gamma$-ray emission by the superposition of pulsed radiation from individual pulsars, whereas BS07 and Cheng et al. (2010) explore IC up-scattering by electrons either in the PWNe themselves or electrons that have been re-accelerated in colliding PWNe shocks. Abdo et al. (2009) find that the model of VJ09 is in good agreement with the $\mathrm{GeV}$ properties of the Fermi-LAT source coincident with the GC 47 Tucanae, while the model of BS07 cannot reproduce its spectral shape. More recently, Cheng et al. (2010) have modelled the GeV spectra of 47 Tucanae and Terzan 5 with IC emission.

In the VHE range, these models rely on IC up-scattering of photons, from the cluster as well as the Galactic stellar and cosmic microwave backgrounds, either collectively by electrons leaving the msPSRs (see VJ09) or again by electrons reaccelerated in colliding shocks between PWNe (see BS07). The models of BS07 and VJ09 predict a flux in the VHE range of $\sim 1 \%$ of the Crab flux for reasonable input parameters, similar to the source flux determined in this work. For Terzan 5, Cheng et al. (2010) do not predict significant $\gamma$-ray emission at energies beyond $100 \mathrm{GeV}$. Adopting the model of VJ09 with updated physical parameters for the GC (Lanzoni et al. 2010) and a distance of $5.9 \mathrm{kpc}$, a population of $220 \pm 50 \mathrm{msPSRs}$ for a magnetic field of $10 \mu \mathrm{G}(167 \pm 39 \mathrm{msPSR}$ for $20 \mu \mathrm{G})$ is required to explain the observed luminosity of the VHE $\gamma$-ray source above $1 \mathrm{TeV}$. This agrees well with the number of msPSRs derived in Abdo et al. (2010a) in the $\mathrm{GeV}$ range. The number of required msPSRs is quite insensitive to the magnetic field in the range 10-20 $\mu \mathrm{G}$, although it increases rapidly outside this range (e.g. by $\sim 50 \%$ at $5 \mu \mathrm{G}$, see VJ09). This model neglects $\gamma$-ray emission from outside $r_{\mathrm{h}}$, due to the uncertainties in the diffusion coefficient (and derived residence time) of the VHE electrons in this region, likely to be unusual if the GC is a dwarf galaxy remnant as proposed by Ferraro et al. (2009). The additional interactions included in BS07 result in an extended IC source of $2^{\prime}-3^{\prime}$ radius, in rough agreement with the observed source size. When scaling their predictions for Terzan 5 (their Fig. 7, solid curve, top panel) in the same way as done in Aharonian et al. (2009), for $180 \mathrm{msPSR}$ of individual spin-down power $10^{34} \mathrm{erg} \mathrm{s}^{-1}$, the predicted integral flux is $F(E>440 \mathrm{GeV}) \approx 1 \times 10^{-12} \mathrm{~cm}^{-2} \mathrm{~s}^{-1}$, at the level of the HESS measurement.

The IC emission should be accompanied by synchrotron emission in the X-ray band (Venter et al. 2008). Non-thermal $\mathrm{X}$-ray emission extending beyond $r_{\mathrm{h}}$ has indeed been discovered from Terzan 5 (Eger et al. 2010). The nature of this X-ray emission, apparently centred on the GC, is still unknown and may be unrelated to HESS J1747-248. Based on the energetics a synchrotron origin seems to be preferred (Eger et al. 2010). This interpretation is challenged by the spatial extension and the 
indication of an offset of the VHE $\gamma$-ray source from the GC centre. A simple model where the X-ray and VHE $\gamma$-ray emission originates in the same population of electrons cannot produce IC $\gamma$-ray emission displaced from the peak of the stellar photon field at the GC centre as observed.

To conclude, the observed flux - but not the morphology - of the VHE $\gamma$-ray source is reasonably reproduced by the msPSRs scenarios. To explain the morphology, more sophisticated models should be tested including particle trapping and non-uniform diffusion across the GC. Since the optical to near-infrared stellar photon field should be up-scattered by the VHE electrons, Klein-Nishina suppression of the IC process should become significant at multiple $\mathrm{TeV}$ energies. This would cause a steepening in the VHE $\gamma$-ray spectrum, which should therefore not follow a pure power law. Statistics are currently too low to test this possibility.

\subsection{Comparison to other globular clusters}

Under the assumption that the observed VHE $\gamma$-ray source is related to Terzan 5, results from other GCs in the same energy band would be valuable. From the Fermi-LAT results (Abdo et al. 2010a), NGC 6388 seems to host a number of msPSRs comparable to Terzan 5. Its larger radiation field, even at its greater distance, makes it an interesting candidate for VHE $\gamma$-ray studies (Abramowski et al. 2011). However, predictions of VHE $\gamma$-ray fluxes for GCs strongly depend on uncertain parameters like the diffusion coefficient and the cluster magnetic field, as well as on the distance from the GC centre.

Among the other GCs observed in the VHE domain, 47 Tucanae is a very interesting object to compare with Terzan 5. It is located at $4 \mathrm{kpc}$ (closer than Terzan 5) and away from the Galactic plane, thus simplifying observation at all wavelengths. It contains the second largest population (23, Ransom 2008) of individually detected msPSRs among GCs (after Terzan 5 with 33), in agreement with the Fermi-LAT estimate of $33 \pm$ 15 msPSRs (Abdo et al. 2010a). HESS derived a flux upper limit on 47 Tucanae (Aharonian et al. 2009) of $F(E>800 \mathrm{GeV})<$ $6.7 \times 10^{-13} \mathrm{~cm}^{-2} \mathrm{~s}^{-1}$, at $99 \%$ confidence level, for a point-like source. The flux from a point-like source analysis centred on Terzan 5 in the same energy range (see Sect. 2) is below this limit. Given the uncertainties on the correcting factors, e.g. the distance to the GCs and the number of msPSRs, and the actual source morphology, this comparison only illustrates that additional observations of GCs will be required to settle the issue of VHE $\gamma$-ray emission from their core.

\subsection{Hadronic VHE $\gamma$-ray production}

The remnants of a type Ia supernova resulting from the merger of two white dwarfs could produce hadronic CRs (see e.g. the VHE $\gamma$-ray detection of SN 1006 by Acero et al. 2010b). GCs are expected to boost the rate of stellar collisions (Shara \& Hurley 2002; Grindlay et al. 2006).

The energy in hadronic CRs required to explain a VHE $\gamma$-ray source of luminosity $L_{\gamma}$ with a hadronic scenario is $E_{\mathrm{pp}}=L_{\gamma} \tau_{\mathrm{pp}} \eta^{-1}(1+S)$, where $\tau_{\mathrm{pp}}$ is the cooling time due to inelastic p-p collisions (linearly dependent on the density $n$ of the ambient medium) and $\eta \approx 1 / 3$ is the fraction of CR energy that is converted into $\pi^{0}$ mesons. CRs below the energy range probed by the observed VHE photons are accounted for by $S$. Here, the HESS observation threshold of $440 \mathrm{GeV}$ translates into a minimum CR energy of $5 \mathrm{TeV}$. With no indication of molecular material at the location of Terzan 5 (Dame et al. 2001), $n \approx 0.1 \mathrm{~cm}^{-3}$ is assumed (following Dickey \& Lockman 1992). For a typical SNR CR spectrum below $5 \mathrm{TeV}$ of spectral index 2.0, Atoyan et al. (2006) derive $S \approx 5$, giving $E_{\mathrm{pp}} \approx 10^{51}\left(n / 0.1 \mathrm{~cm}^{-3}\right)^{-1}(d / 5.9 \mathrm{kpc})^{2} \mathrm{erg}$, which is somewhat high for a supernova.

An alternative scenario may be provided by the remnant of a short gamma-ray burst (GRB) induced by the merger of two neutron stars, see e.g. Nakar (2007) for a review and Grindlay et al. (2006) in the context of GCs. In relativistic shocks, the transfer of the initial kinetic energy to CRs is expected to be very efficient (Atoyan et al. 2006), so the kinetic energy of a short GRB could roughly provide the energy in CRs needed for a hadronic interpretation of the HESS source. Such a GRB remnant would be spatially extended, owing to the diffusion of CRs away from the explosion site, in relation to its age $t \approx r^{2} / 2 D$, where $r$ is the radius of the remnant and $D$ the diffusion coefficient.

Assuming CRs of energy $5 \mathrm{TeV}$, the extension of the present source would give $t \approx 10^{3}$ yr for $D=10^{28} \mathrm{~cm}^{2} \mathrm{~s}^{-1}$ (in the Galactic disk, Atoyan et al. 2006). The rate of short GRBs in the Galaxy should be about one event every $10^{4}\left(f_{\mathrm{b}}^{-1} / 100\right)^{-1} \mathrm{yr}$, where $f_{\mathrm{b}}$ is the GRB beaming factor, uncertain but expected to fall in the range $1 \ll f_{\mathrm{b}}^{-1}<100$ (Nakar 2007). This estimate is based on a rate of short GRBs of $10 \mathrm{Gpc}^{-3} \mathrm{yr}^{-1}$ (Nakar 2007) and a density of Milky Way-type galaxies in the local Universe of $10^{-2}$ galaxies per $\mathrm{Mpc}^{-3}$ (Cole et al. 2001). For $D$ below $10^{27} \mathrm{~cm}^{2} \mathrm{~s}^{-1}$ in the $\mathrm{TeV}$ range in the vicinity of the GC, as suggested by Crocker \& Aharonian (2011), the age obtained from the source extension is roughly compatible with the rate of short GRBs in the Galaxy. Nevertheless, the value of $D$ in this region may differ significantly from the available estimates.

A hadronic scenario could accommodate the observed pure power-law spectrum in the VHE $\gamma$-ray domain, as opposed to a leptonic scenario. Detection of thermal X-rays would strengthen the case for a GRB remnant, where shocks driven by subrelativistic ejecta expelled during the merger would heat the interstellar medium (Domainko \& Ruffert 2005, 2008). However, no observational evidence was found in support of either the supernova or GRB remnant scenario.

\section{Outlook}

The nature of HESS J1747-248 is uncertain, since no counterpart or model can fully explain the observed morphology. An association with Terzan 5 is tantalizing, but the available data do not firmly prove this scenario.

Several tests could be done to clarify the nature of the VHE source. In the X-ray range, a large FoV and an extended energy range would be desirable. XMM-Newton with its large FoV is suitable for searching for counterparts over the whole extent of HESS J1747-248. As a complement, the upcoming NuSTAR mission (Harrison et al. 2010) will provide significantly improved sensitivity in the $\sim 10-70 \mathrm{keV}$ range, which is less affected by neutral hydrogen absorption. This may help in constraining the nature of the diffuse X-ray emission found by Chandra. Pulsar searches, with Fermi-LAT and radio telescopes, may reveal a powerful pulsar in the vicinity of HESS J1747-248. Finally H.E.S.S phase II, which will extend observations in the VHE $\gamma$-ray range to lower energies, will further characterize the source.

In parallel, more sophisticated source models might narrow down the list of applicable emission scenarios.

Acknowledgements. The support of the Namibian authorities and of the University of Namibia in facilitating the construction and operation of H.E.S.S is gratefully acknowledged, as is the support by the German Ministry for Education and Research (BMBF), the Max Planck Society, the French Ministry for Research, the CNRS-IN2P3 and the Astroparticle Interdisciplinary 
Programme of the CNRS, the U.K. Science and Technology Facilities Council (STFC), the IPNP of the Charles University, the Polish Ministry of Science and Higher Education, the South African Department of Science and Technology and National Research Foundation, and by the University of Namibia. We appreciate the excellent work of the technical support staff in Berlin, Durham, Hamburg, Heidelberg, Palaiseau, Paris, Saclay, and in Namibia in the construction and operation of the equipment.

\section{References}

Abdo, A. A., Ackermann, M., Ajello, M., et al. (The Fermi LAT Collaboration) 2009, Science, 325, 845

Abdo, A. A., Ackermann, M., Ajello, M., et al. (The Fermi LAT Collaboration) 2010a, A\&A, 524, 75

Abdo, A. A., Ackermann, M., Ajello, M., et al. (The Fermi LAT Collaboration) 2010b, ApJS, 187, 460

Abramowski, A., Acero, F., Aharonian, F., et al. (H.E.S.S. Collaboration) 2011, ApJ, 735, 12

Acero, F., Aharonian, F., Akhperjanian, A. G., et al. (H.E.S.S. Collaboration) 2010a, MNRAS, 402, 1877

Acero, F., Aharonian, F., Akhperjanian, A. G., et al. (H.E.S.S. Collaboration) 2010b, A\&A, 516, A62

Aharonian, F., Akhperjanian, A. G., Bazer-Bachi, A. R., et al. (H.E.S.S. Collaboration) 2006, A\&A, 457, 899

Aharonian, F. A., Akhperjanian, A. G., Anton, G., et al. 2009, A\&A, 499, 273

Anderhub, H., Antonelli, L. A., Antoranz, P., et al. (MAGIC Collaboration) 2009, ApJ, 702, 266

Atoyan, A., Buckley, J., \& Krawczynski, H. 2006, ApJ, 642, L153

Bednarek, W., \& Sitarek, J. 2007, MNRAS, 377, 920

Berge, D., Funk, S., \& Hinton, J. 2007, A\&A, 466, 1219

Camilo, F., \& Rasio, F. A. 2005, ASPC, 328, 147

Carrigan, S., Hinton, J. A., Hofmann, W., et al. (H.E.S.S. Collaboration) 2008, Proc. 30th ICRC. Mexico, 2, 659

Chaves, R. C. G. (H.E.S.S. Collaboration) 2009 [arXiv: 0907.0768v1]

Cheng, K. S., Chernyshov, D. O., Dogiel, V. A., Hui, C. Y., \& Kong, A. K. H. 2010, ApJ, 723, 1219

Clapson, A.-C., Domainko, W., Jamrozy, M., et al. 2011, A\&A, accepted [arXiv: 1105. 5579]

Cole, S., Norberg, P., Baugh, C. M., et al. 2001, MNRAS, 326, 255

Crocker, R. M., \& Aharonian, F. A. 2011, Phys. Rev. Lett., 106, 101102

McCutcheon, M. (VERITAS Collaboration) 2010 [arXiv: 0907 .4974]

Dame, T. M., Hartmann, D., \& Thaddeus, P. 2001, ApJ, 547, 792

Dickey, J. M., \& Lockman, F. J. 1990, ARA\&A, 28, 215

Domainko, W., \& Ruffert, M. 2005, A\&A, 444, L33

Domainko, W., \& Ruffert, M. 2008, AdSpR, 41, 518

Eger, P., Domainko, W., \& Clapson, A.-C. 2010, A\&A, 513, 66

Feldman, G. J., \& Cousins, R. D. 1998, Phys. Rev. D, 57, 3873

Ferraro, F. R., Beccari, G., Dalessandro, E., et al. 2009, Nature, 462, 1028

Freeman, P., Doe, S., \& Siemiginowska, A. 2001, SPIE, 4477, 76

Fruchter, A. S., \& Goss, W. M. 2000, ApJ, 536, 865

Grindlay, J., Portegies, Z. S., \& McMillan, S. 2006, Nat. Phys., 2, 116

Gonthier, P. L., Van Guilder, R., \& Harding, A. K. 2004, ApJ, 604775

Harrison, F. A., Boggs, S., Christensen, F., et al. 2010, SPIE, 7732, 21

Kong, A. K. H., Hui, C. Y., \& Cheng, K. S. 2010, ApJ, 712, 36

Lanzoni, B., Ferraro, F. R., Dalessandro, E., et al. 2010, ApJ, 717, 653

Manchester, R. N., Hobbs, G. B., Teoh, A., \& Hobbs, M. 2005, AJ, 129, 1993

Mattana, F., Falanga, M., Götz, D., et al. 2009, ApJ, 694, 12

Nakar, E. 2007, PhR, 442, 166

de Naurois, M., \& Rolland, L. 2009, APh, 32, 231

Ohm, S., van Eldik, C., \& Egberts, K. 2009, APh, 31, 383

Pooley, D., \& Hut, P. 2006, ApJ, 646, L143

Ransom, S. M. 2008, in IAU Symp. 246, ed. E. Vesperini, M. Giersz, \& A. Sills, 291

Rowell, G. P. 2003, A\&A, 410, 389

Shara, M. M., \& Hurley, J. R. 2002, ApJ, 571, 830

Tam, P. H. T., Kong, A. K. H., Hui, C. Y., et al. 2011, ApJ, 729, 90

Venter, C., \& de Jager, O. C. 2008, AIPC, 1085, 277

Venter, C., de Jager, O. C., \& Clapson, A.-C. 2009, ApJ, 696, L52

1 Universität Hamburg, Institut für Experimentalphysik, Luruper Chaussee 149, 22761 Hamburg, Germany

2 Laboratoire Univers et Particules de Montpellier, Université Montpellier 2, CNRS/IN2P3, CC 72, Place Eugène Bataillon, 34095 Montpellier Cedex 5, France
3 Max-Planck-Institut für Kernphysik, PO Box 103980, 69029 Heidelberg, Germany

e-mail: [clapson; domainko] @mpi-hd.mpg . de

4 Dublin Institute for Advanced Studies, 31 Fitzwilliam Place, Dublin 2, Ireland

5 National Academy of Sciences of the Republic of Armenia, Yerevan

6 Yerevan Physics Institute, 2 Alikhanian Brothers St., 375036 Yerevan, Armenia

7 Universität Erlangen-Nürnberg, Physikalisches Institut, Erwin-Rommel-Str. 1, 91058 Erlangen, Germany

8 Nicolaus Copernicus Astronomical Center, ul. Bartycka 18, 00-716 Warsaw, Poland

9 CEA Saclay, DSM/IRFU, 91191 Gif-Sur-Yvette Cedex, France

10 University of Durham, Department of Physics, South Road, Durham DH1 3LE, UK

11 Astroparticule et Cosmologie (APC), CNRS, Université Paris 7 Denis Diderot, 10 rue Alice Domon et Léonie Duquet, 75205 Paris Cedex 13, France (UMR 7164: CNRS, Université Paris VII, CEA, Observatoire de Paris)

12 Laboratoire Leprince-Ringuet, Ecole Polytechnique, CNRS/IN2P3, 91128 Palaiseau, France

13 Institut für Theoretische Physik, Lehrstuhl IV: Weltraum und Astrophysik, Ruhr-Universität Bochum, 44780 Bochum, Germany

14 Landessternwarte, Universität Heidelberg, Königstuhl, 69117 Heidelberg, Germany

15 Institut für Physik, Humboldt-Universität zu Berlin, Newtonstr. 15, 12489 Berlin, Germany

16 LUTH, Observatoire de Paris, CNRS, Université Paris Diderot, 5 place Jules Janssen, 92190 Meudon, France

17 LPNHE, Université Pierre et Marie Curie Paris 6, Université Denis Diderot Paris 7, CNRS/IN2P3, 4 place Jussieu, 75252, Paris Cedex 5, France

18 Institut für Astronomie und Astrophysik, Universität Tübingen, Sand 1, 72076 Tübingen, Germany

19 Astronomical Observatory, The University of Warsaw, Al. Ujazdowskie 4, 00-478 Warsaw, Poland

20 Unit for Space Physics, North-West University, Potchefstroom 2520, South Africa

21 Laboratoire d'Annecy-le-Vieux de Physique des Particules, Université de Savoie, CNRS/IN2P3, 74941 Annecy-le-Vieux, France

22 Oskar Klein Centre, Department of Physics, Stockholm University, Albanova University Center, 10691 Stockholm, Sweden

23 University of Namibia, Department of Physics, Private Bag 13301, Windhoek, Namibia

24 Laboratoire d'Astrophysique de Grenoble, INSU/CNRS, Université Joseph Fourier, BP 53, 38041 Grenoble Cedex 9, France

25 Department of Physics and Astronomy, The University of Leicester, University Road, Leicester, LE1 7RH, UK

26 Instytut Fizyki Ja̧drowej PAN, ul. Radzikowskiego 152, 31-342 Kraków, Poland

27 Institut für Astro- und Teilchenphysik, Leopold-FranzensUniversität Innsbruck, 6020 Innsbruck, Austria

28 Obserwatorium Astronomiczne, Uniwersytet Jagielloński, ul. Orla 171, 30-244 Kraków, Poland

29 Toruń Centre for Astronomy, Nicolaus Copernicus University, ul. Gagarina 11, 87-100 Toruń, Poland

30 School of Chemistry \& Physics, University of Adelaide, Adelaide 5005, Australia

31 Charles University, Faculty of Mathematics and Physics, Institute of Particle and Nuclear Physics, V Holešovičkách 2, 18000 Prague 8, Czech Republic

32 School of Physics \& Astronomy, University of Leeds, Leeds LS2 9JT, UK

33 European Associated Laboratory for Gamma-Ray Astronomy, jointly supported by CNRS and MPG

34 Oskar Klein Centre, Department of Physics, Royal Institute of Technology (KTH), Albanova, 10691 Stockholm, Sweden 